\title{
Abundancia de garcilla bueyera Bubulcus ibis L. 1758 en Cantabria durante el periodo 2012-2015.
}

\section{Abundance of the cattle egret Bubulcus ibis L. 1758 in Cantabria during 2012-2015.}

Ludovico de Vega del Val ${ }^{1}$, Aitor Sanz-Gil ${ }^{2}$

\section{Resumen}

La garcilla bueyera (Bubulcus ibis L.) ha experimentado una gran expansión a nivel global en los últimos 100 años, llegando a colonizar la mayor parte del planeta a excepción del continente Antártico. Como especie nueva en Cantabria, el objetivo principal de este trabajo ha sido conocer su fenología y abundancia. En primer lugar se localizaron los dormideros, un total de 18, situados a lo largo de la mitad norte de la provincia, la mayoría en humedales de la franja costera. Posteriormente se realizaron los censos, siempre al atardecer, cuando las garcillas acuden a estos emplazamientos para pernoctar. Los resultados obtenidos indican una mayor abundancia y distribución de ejemplares durante el invierno (más de 6.000 individuos) que en la época estival (menos de 4.000), cuando más del $90 \%$ de los ejemplares se concentra en las dos colonias de cría existentes. Existe una alta movilidad de la especie, ya que sin que apenas cambie la abundancia global encontramos que la abundancia de ejemplares dentro de cada dormidero es muy variable. Por otro lado, entre los dos dormideros principales más lejanos hay tan solo 75 km en línea recta, una distancia similar a lo que sabemos puede recorrer una garcilla bueyera diariamente $(60-70 \mathrm{~km})$. Con respecto al uso que hacen de los dormideros, se han registrado diferentes niveles de ocupación y fidelidad, que van desde los más de mil ejemplares pernoctando durante al menos dos meses seguidos, hasta aquellas localizaciones en las que aparecen menos de cien aves de manera puntual y su presencia no vuelve a registrarse en los meses posteriores. Podemos concluir que esta especie se ha consolidado como residente en

\footnotetext{
1 Técnico Ambiental en el P. N. Marismas de Santoña, Victoria y Joyel. Cantabria

*Correspondencia: Iudovicodevega@hotmail.com
}

2 Licenciado Ciencias Ambientales

*Correspondencia: aitorsanzgil@gmail.com 
Cantabria tras su reciente aparición hace poco más de veinte años, mostrando una gran capacidad a la hora de asentarse en el territorio y encontrar lugares de descanso y reproducción.

Palabras clave: Garcilla bueyera, Bubulcus ibis, dormidero, población, expansión.

\begin{abstract}
The range of the cattle egret has expanded greatly over the last 100 years, leading to its colonization of most of the planet, with the exception of the Antarctic. As a new species in Cantabria, the main objective of this study was to gather information on its abundance and phenology. Firstly, a total of 18 roosts were found in the northern half of the province, the majority of which were located within the coastal belt and near-shore wetlands. Later, a census was carried out at dusk, when the cattle egrets returned to their roosts to spend the night. The results obtained indicate a greater abundance and distribution of individuals during the winter months (more than 6,000 individuals) than in the summer period (less than 4,000 individuals), when more than $90 \%$ of the birds are concentrated within the two established breeding colonies. The species exhibits a high level of mobility, as, without significant changes in the overall population, we found that the cattle egrets' presence/absence in roosts is highly variable. Moreover, between the two main roosts there are only $75 \mathrm{~km}$, as the crow flies, which is the known distance that this species can fly on a daily basis (60-70 km) (Cramp y Simmons, 1977). With respect to the use made of roosts, different levels of occupation and fidelity have been detected, ranging from more than a thousand individuals spending the night during at least two consecutive months, to those locations visited on a sporadic basis by less than one hundred birds, the presence of which is not recorded in subsequent months. Despite the fact that this species has been a resident of Cantabria for only 20 years, this study demonstrates its great capacity to occupy new territory, feed, rest and breed.
\end{abstract}

Key words: Cattle egret, Bubulcus ibis, roost, population, expansion.

\title{
Laburpena
}

Lertxuntxo itzainak (Bubulcus ibis L.) zabalkunde itzela izan du mundu mailan azken 100 urteetan, Antartikoa izan ezik planetako toki gehienak kolonizatzera iritsi delarik. Kantabrian espezie berria izanik, lan honen helburu nagusia izan da espezie honen fenologia eta ugaritasuna jakitea. Lehen urratsa espeziaren gaueko lekuak aurkitzea izan da, 18 guztira, probintziaren erdialdetik iparralderantz kokatuak, gehienak kostaldeko hezeguneetan. Ondoren erroldak egin dira, ilunabarretan beti ere, lertxuntxoak gaua igarotzeko biltzen direnean. Jasotako emaitzek erakusten dutenez, aleen kopurua eta banaketa neguan da handiena (6.000 ale baino gehiago), uda garaian baino (4.000 baino gutxiago), sasoi horretan aleen $\% 90$ baino gehiago bi ugaltze koloniatan biltzen direlarik. Mugikortasun handia erakutsi du espezieak, zeren eta kopuru orokorrean ia aldaketarik izan gabe, gau lekuetan ale kopuruak oso aldakorrak baitira. Bestalde, bi gau leku urrunenen arteko distantzia soilik 75 km-koa da zuzenean, lertxuntxo itzainak egunean egin dezakeen distantziaren parekoa (60-70 km). Eta gaueko leku horiek nola erabiltzen dituzten, horretan okupazio eta fideltasun maila desberdinak ikusi dira; kasurako, 1.000 alek elkarrekin bi hilabetez jarraian gaua eman dutenekoa, edota modu puntualean 100 ale baino 
gutxiagoko gau lekuak, eta ale horien berragerpenik gabe hurrengo hiletan. Horrenbestez, esan daiteke ezen Lertxuntxo itzaina, Kantabrian, bertako espezie bilakatu dela, soilik 20 urte lehenago agertu ostean, ahalmen handia erakutsi duelarik bai lurraldean finkatzeko eta bai atseden hartzeko nahiz ugaltzeko gune aproposak topatzeko.

Gako hitzak: Lertxuntxo itzaina, Bubulcus ibis, gaueko leku, populazio hedatzailea.

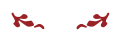

\section{Introducción}

La garcilla bueyera Bubulcus ibis es originaria de las zonas tropicales y subtropicales de África y Asia así como del suroeste Europeo (sur de España y Portugal), donde se conoce su presencia en los siglos XVI y XVII (Guardiola Gómez, 1999). Posteriormente sufrió un fuerte declive en sus áreas de cría europeas, del que se recuperó a lo largo del siglo XX, momento en el que empieza a ocupar rápidamente nuevos territorios: sur de Francia en 1958, norte de Francia en 1981 e Italia en 1985 (Martínez-Vilalta y Motis, 1992). En la actualidad está presente en todos los continentes a excepción de la Antártida, ya que desde finales del siglo XIX y a lo largo del XX experimentó una gran expansión a nivel mundial (Del Hoyo et al. 1992; Martínez-Vilalta y Motis, 1992). Más recientemente se ha confirmado su reproducción en Reino Unido (Nightingale y Dempsey, 2008) y también en Irlanda (Barret, 2008). Fuera de Europa, comenzó su expansión por el continente africano y llegó a América del Sur a finales del siglo XIX, citándose en Guayana francesa y Surinam ya en 1877 (Krebs et al., 1994). Tras haber cruzado el océano atlántico continuó expandiendo su área de distribución por América Central y Estados Unidos, a donde llega entre los años 30 y 40 del siglo pasado. La especie fue citada por primera vez como reproductora en Florida en 1953 y en Canadá en 1962 (Martínez-Vilalta y Motis, 1992).

En la península Ibérica, a comienzos del siglo pasado la garcilla bueyera se encontraba únicamente en la zona oeste de Andalucía y sur de Portugal, así como en el entorno del Parque Nacional de Doñana, no siendo hasta la década de los 60 del pasado siglo cuando la especie muestra signos de expansión (De Juana y García, 2015). En la actualidad se encuentra ampliamente distribuida en la mitad sur peninsular y el cuadrante nororiental durante la invernada, mientras que en época de cría, las colonias parecen concentrarse en el cuadrante suroccidental y costa del mediterráneo (Garrido et al., 2012).

La población reproductora de Cantabria es por tanto, la única en toda la franja costera cantábrica y parece tener su origen en un grupo de aves asilvestradas procedentes del zoológico de Santillana del Mar, a donde llegó en 1990 (Bahillo y Alonso, 1998). A partir de entonces comenzaron a observarse pequeños grupos de individuos por las praderías de casi todo el occidente cántabro. Posteriormente también podrían haber llegado ejemplares a través del valle del Ebro en sus movimientos de dispersión desde el sur peninsular (Sarasa et al., 1997; Serradilla et al., 2011). 
Debido a lo novedoso de su presencia en territorios cántabros, la información disponible de la especie en la zona se limitaba a algunos censos puntuales sin continuidad en el tiempo. El que se presenta a continuación es, hasta donde nosotros sabemos, el primer estudio continuado de garcillas bueyeras en la provincia de Cantabria. En él, se describe la evolución a lo largo del año de la abundancia y la distribución de los dormideros durante el periodo 2012-2015 obteniendo así un patrón fenológico de la especie en la región. Por otro lado se presentan datos actualizados sobre las colonias de cría, su ubicación y número de parejas reproductoras en el territorio cántabro.

\section{Área de estudio y métodos}

Con 5.321 km², Cantabria se ubica en la costa norte de la Península Ibérica y limita con otras tres comunidades autónomas a saber: País Vasco por el este, Asturias por el oeste y Castilla y León al sur. El clima, de tipo atlántico, es templado y húmedo, lo que se traduce en inviernos y veranos sin temperaturas extremas y abundantes precipitaciones a lo largo del año.

Como parte del trabajo, los dormideros fueron localizados en el año 2011, previamente al comienzo de los censos. Dichos dormideros fueron censados una vez al mes en 2013 y 2014, así como en enero de 2012 y 2015, para obtener datos de aves invernantes. Los conteos se llevaron a cabo mediante observación directa con la ayuda de binoculares 10×42. Éstos se realizaron siempre al atardecer, momento en el que las garcillas se congregan para pasar la noche. Fue necesario por tanto, situarse en el punto de censo en torno a una hora y media antes de la ausencia de luz (unos 15-20 minutos tras la puesta de sol), momento en el que cesa la entrada de individuos al dormidero. El equipo de censo estuvo formado por tres personas. Una de ellas se encargó de los dos dormideros situados en la zona más occidental (Comillas y San Vicente de la Barquera) mientras que el resto de ubicaciones fueron censadas por los otros dos componentes y autores del trabajo (en ningún caso se realizaron censos simultáneos sobre el mismo dormidero). Para evitar posibles errores, ya que la mayoría de dormideros se encuentran próximos entre sí y por tanto podían ser utilizados por las garcillas indistintamente, se realizaron censos simultáneos, con un censador en cada uno de ellos. Los bandos fueron registrados contabilizando los ejemplares uno a uno, ya que en la zona de estudio la mayoría de ellos no superaban el centenar de ejemplares y podían, por tanto, ser contados con exactitud por censadores experimentados. En las escasas ocasiones en las que los bandos superaron con creces el centenar de ejemplares, se hizo una estima de su número, ya que la rapidez con la que las garcillas Ilegaban y se posaban junto a las demás en el dormidero hacía inviable el conteo individual. En función de la fidelidad, los dormideros se consideraron "principales" cuando fueron ocupados durante al menos dos meses consecutivos, sumando como mínimo cinco meses durante el periodo 2013-2014. Por el contrario, como dormideros "secundarios" se consideraron aquellos ocupados por un periodo no superior a los dos meses consecutivos, o bien, aun 
superando este tiempo, no sumaron más de unas pocas decenas de aves.

Para cada dormidero se anotó además el hábitat (marisma, ría, laguna dulce etc.) y el sustrato (árboles, arbustos, carrizal, etc.) en el que se emplazaba.

Por último, se tomaron datos de la llegada del primer y último bando, tamaño de los mismos y cómo y dónde se situaban las aves al llegar, en busca de información sobre patrones de conducta de las garcillas en sus dormideros.

\section{Resultados}

Se localizaron 18 dormideros situados todos ellos entre las localidades de Limpias y San Vicente de la Barquera (Figura 1), de modo que entre las dos localizaciones más alejadas existen 78 km en línea recta. De ellos, 10 fueron clasificados como dormideros principales

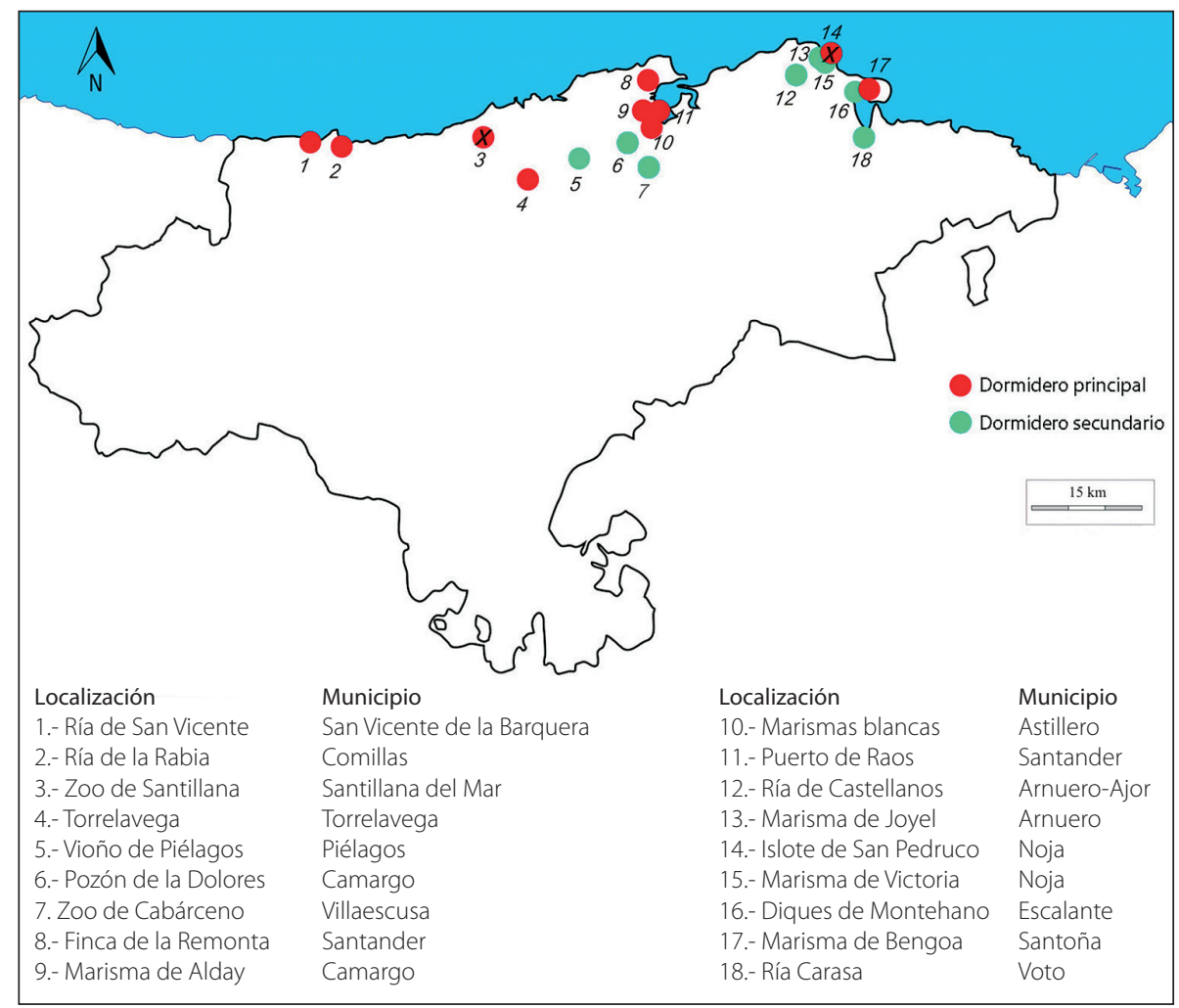

Fig. 1.- Localización de dormideros de garcilla bueyera en Cantabria durante el periodo 2012-2015. Marcadas con una $X$ se señalan las dos colonias de cría existentes ( $n^{\circ} 3$ y 14 ).

Fig. 1.- Location of cattle egret roosts in Cantabria during the period 2012-2015. The two breeding colonies (nos. 3 and 14) are marked with an X. 
y 8 como secundarios (Tablas 1 y 2). Tan solo en San Pedruco (Noja), el dormidero fue utilizado durante un año completo (año 2014), mientras que, por el contrario, en algunos dormideros secundarios tan solo se detectó la presencia de garcillas un único día a lo largo de cinco días de censos consecutivos.

Según los resultados expresados en las tablas 1 y 2 y los varemos utilizados para la distinción entre dormideros principales o secundarios existen dos excepciones. Se trata de los dormideros del puerto de Raos y la finca de la Remonta, ambos en Santander. Estos han sido catalogados como "principales" ya que durante los años 2011 y 2012, periodo en el que se localizaron los dormideros y se realizaron los primeros censos, las dos ubicaciones fueron ocupadas por las garcillas durante más de dos meses, superándose en ocasiones el millar de individuos, aunque no haya sido así en los años 2013 y 2014.

El número de garcillas en cada dormidero según los censos mensuales realizados en el periodo 2013-2014 se expone en las Tablas 1 y 2. El número total de ejemplares fue mínimo $(\bar{\chi}=3.760,4 \pm 263,6$ (EEM) aves) en el periodo reproductor (marzo-julio) y máximo durante el paso migratorio postnupcial y la invernada (octubre-febrero) $(\bar{\chi}=6.800,4 \pm 139,5$ (EEM) aves) (Figura 2). Con respecto al censo del mes de enero en el periodo 2012-2015, el número medio de garcillas bueyeras se sitúa en algo menos de 7.000 individuos $(\bar{\chi} .=6.856,5$ $\pm 283,16$ (EEM)), si bien en 2014 hubo un pico de abundancia, alcanzándose el máximo absoluto en el periodo de estudio (7.606 ejemplares).

Con estos datos podemos establecer un patrón fenológico de la especie en la provincia, donde, en base a la presencia de individuos durante todo el año y al registro de un ejemplar anillado en la colonia de Noja (observado en el mes de noviembre de 2014 en el dormidero de Bengoa, en el pueblo de Santoña) parece existir una fracción de la población con carácter sedentario y otra con carácter invernante. Aquellas garcillas que permanecen durante todo el año, inician su etapa reproductora a finales de marzo, extendiéndose ésta hasta principios de agosto en el área de estudio. En estas fechas, más del 90\% de las garcillas se encuentran concentradas en las colonias de cría existentes. Los efectivos aumentan ya a partir de agosto, probablemente con la incorporación de los pollos del año, aumento que continúa de manera paulatina durante los meses siguientes hasta noviembre, cuando se produce un pico de máxima abundancia, para descender en el mes de diciembre y volver a aumentar en enero, dando un segundo pico de abundancia.

En febrero, con el final del invierno, de nuevo disminuyen los efectivos, con la partida de los primeros individuos rumbo a las áreas de reproducción. En el año 2013 se observó un descenso continuado hasta el mes de julio, dándose el mínimo absoluto con tan solo 2.485 ejemplares, mientras que en 2014 la abundancia de garcillas se estabilizó en primavera, de modo que entre marzo y julio el resultado de los censos no sufrió grandes variaciones.

En la actualidad existen en la provincia únicamente dos colonias de cría: una en Santillana del Mar, que alberga 350-400 parejas (censo de 2014) y otra en el islote de San Pedruco (Noja), en la que se censaron 450 parejas en 2014 y 500 en 2015. Las colonias se encuentran separadas por unos $48 \mathrm{~km}$, una en la costa oriental y otra en la occidental, de manera que 


\begin{tabular}{|l|c|c|c|c|c|c|c|c|c|}
\hline \multicolumn{10}{|c|}{ DORMIDEROS PRINCIPALES } \\
\hline 2013 & $\begin{array}{c}\text { San } \\
\text { Pedruco }\end{array}$ & $\begin{array}{c}\text { M. } \\
\text { Bengoa }\end{array}$ & $\begin{array}{c}\text { M. } \\
\text { Blancas }\end{array}$ & $\begin{array}{c}\text { M. } \\
\text { de Alday }\end{array}$ & $\begin{array}{c}\text { Zoo } \\
\text { Santillana }\end{array}$ & $\begin{array}{c}\text { Torre- } \\
\text { lavega }\end{array}$ & $\begin{array}{c}\text { S. } \\
\text { Vicente }\end{array}$ & Comillas & TOTAL \\
\hline ENERO & 0 & 1450 & 0 & 3116 & 0 & 2000 & 43 & 24 & 6633 \\
FEBRERO & 0 & 0 & 0 & 3900 & 0 & 1770 & 132 & 45 & 5847 \\
MARZO & 0 & 800 & 0 & 1718 & 100 & 1803 & 101 & 26 & 4548 \\
ABRIL & 1050 & 800 & 0 & 375 & 2350 & 538 & 11 & 30 & 5154 \\
MAYO & 1050 & 85 & 0 & 0 & 2400 & 272 & 3 & 13 & 3823 \\
JUNIO & 1223 & 150 & 0 & 0 & 1700 & 0 & 0 & 0 & 3073 \\
JULIO & 679 & 500 & 0 & 412 & 885 & 0 & 9 & 0 & 2485 \\
AGOSTO & 515 & 1100 & 2500 & 430 & 650 & 466 & 37 & 0 & 5698 \\
SEPTIEMBRE & 597 & 1411 & 3015 & 0 & 0 & 686 & 145 & 0 & 5854 \\
OCTUBRE & 600 & 1574 & 3080 & 0 & 0 & 986 & 271 & 5 & 6516 \\
NOVIEMBRE & 565 & 1625 & 3671 & 0 & 0 & 1005 & 150 & 120 & 7136 \\
DICIEMBRE & 515 & 0 & 3161 & 931 & 0 & 1701 & 158 & 0 & 6466 \\
\hline
\end{tabular}

\begin{tabular}{|l|c|c|c|c|c|c|c|}
\hline \multicolumn{8}{|c|}{ DORMIDEROS SECUNDARIOS } \\
\hline 2013 & Carasa & $\begin{array}{c}\text { Ría } \\
\text { Venera }\end{array}$ & M. Joyel & $\begin{array}{c}\text { Monte- } \\
\text { hano }\end{array}$ & $\begin{array}{c}\text { Pozon. } \\
\text { Camargo }\end{array}$ & $\begin{array}{c}\text { Vioño de } \\
\text { Piélagos }\end{array}$ & TOTAL \\
\hline ENERO & 20 & 145 & 0 & 0 & 0 & 32 & 197 \\
FEBRERO & 0 & 78 & 477 & 36 & 0 & 11 & 602 \\
MARZO & 0 & 0 & 550 & 0 & 0 & 13 & 563 \\
ABRIL & 0 & 0 & 0 & 0 & 0 & 0 & 0 \\
MAYO & 0 & 0 & 0 & 0 & 0 & 0 & 0 \\
JUNIO & 0 & 0 & 0 & 0 & 0 & 0 & 0 \\
JULIO & 0 & 0 & 0 & 0 & 0 & 0 & 0 \\
AGOSTO & 170 & 0 & 0 & 0 & 0 & 0 & 170 \\
SEPTIEMBRE & 0 & 0 & 0 & 0 & 0 & 0 & 0 \\
OCTUBRE & 0 & 0 & 0 & 0 & 38 & 0 & 38 \\
NOVIEMBRE & 0 & 80 & 0 & 14 & 79 & 54 & 227 \\
DICIEMBRE & 0 & 0 & 0 & 0 & 0 & 26 & 26 \\
\hline
\end{tabular}

Tabla 1.- Censo mensual de garcilla bueyera en los dormideros de Cantabria durante 2013.

Table 1.- Monthly census of the cattle egret in roosts located in Cantabria during 2013. 


\begin{tabular}{|l|c|c|c|c|c|c|c|c|c|c|}
\hline \multicolumn{10}{|c|}{ DORMIDEROS PRINCIPALES } \\
\hline 2014 & $\begin{array}{c}\text { San } \\
\text { Pedruco }\end{array}$ & $\begin{array}{c}\text { M. } \\
\text { Bengoa }\end{array}$ & $\begin{array}{c}\text { M. } \\
\text { Blancas }\end{array}$ & $\begin{array}{c}\text { La } \\
\text { Remonta }\end{array}$ & $\begin{array}{c}\text { Puerto } \\
\text { Raos }\end{array}$ & $\begin{array}{c}\text { Zoo- } \\
\text { Santillana }\end{array}$ & $\begin{array}{c}\text { Torre- } \\
\text { lavega }\end{array}$ & $\begin{array}{c}\text { San } \\
\text { Vicente }\end{array}$ & Comillas & TOTAL \\
\hline ENERO & 514 & 860 & 4150 & 0 & 0 & 0 & 1882 & 179 & 0 & 7585 \\
FEBRERO & 40 & 0 & 3883 & 0 & 0 & 0 & 1538 & 187 & 0 & 5648 \\
MARZO & 839 & 0 & 1302 & 0 & 0 & 0 & 1680 & 163 & 0 & 3984 \\
ABRIL & 1761 & 0 & 0 & 0 & 0 & 1500 & 0 & 114 & 0 & 3375 \\
MAYO & 1812 & 0 & 0 & 0 & 0 & 1537 & 0 & 49 & 0 & 3398 \\
JUNIO & 1783 & 0 & 0 & 0 & 0 & 1841 & 0 & 0 & 0 & 3624 \\
JULIO & 907 & 337 & 75 & 0 & 0 & 1956 & 0 & 297 & 0 & 3572 \\
AGOSTO & 605 & 450 & 1947 & 0 & 0 & 1900 & 0 & 288 & 0 & 5190 \\
SEPTIEMBRE & 609 & 544 & 2100 & 900 & 0 & 397 & 0 & 291 & 0 & 4841 \\
OCTUBRE & 606 & 1661 & 1775 & 897 & 105 & 0 & 455 & 0 & 264 & 5763 \\
NOVIEMBRE & 806 & 826 & 804 & 1752 & 488 & 0 & 1263 & 0 & 226 & 6165 \\
DICIEMBRE & 1012 & 0 & 913 & 1859 & 508 & 0 & 1891 & 0 & 185 & 6368 \\
\hline
\end{tabular}

\begin{tabular}{|l|c|c|c|c|c|c|c|}
\hline \multicolumn{7}{|c|}{ DORMIDEROS SECUNDARIOS } \\
\hline 2014 & Carasa & $\begin{array}{c}\text { Ría } \\
\text { Venera }\end{array}$ & M. Joyel & $\begin{array}{c}\text { M.- } \\
\text { Victoria }\end{array}$ & $\begin{array}{c}\text { Zoo } \\
\text { Cabárceno }\end{array}$ & $\begin{array}{c}\text { Vioño de } \\
\text { Piélagos }\end{array}$ & TOTAL \\
\hline ENERO & 0 & 0 & 0 & 0 & 0 & 21 & 21 \\
FEBRERO & 106 & 205 & 435 & 0 & 0 & 0 & 746 \\
MARZO & 0 & 0 & 0 & 0 & 0 & 5 & 5 \\
ABRIL & 0 & 0 & 0 & 0 & 0 & 0 & 0 \\
MAYO & 0 & 0 & 0 & 0 & 0 & 0 & 0 \\
JUNIO & 0 & 0 & 0 & 0 & 0 & 0 & 0 \\
JULIO & 0 & 0 & 0 & 0 & 0 & 0 & 0 \\
AGOSTO & 0 & 0 & 0 & 0 & 0 & 0 & 0 \\
SEPTIEMBRE & 0 & 0 & 0 & 0 & 846 & 0 & 846 \\
OCTUBRE & 0 & 13 & 0 & 14 & 915 & 0 & 942 \\
NOVIEMBRE & 0 & 56 & 0 & 37 & 905 & 49 & 1047 \\
DICIEMBRE & 0 & 0 & 0 & 0 & 0 & 31 & 31 \\
\hline
\end{tabular}

Tabla 2.- Censo mensual de garcilla bueyera en los dormideros de Cantabria durante 2014.

Table 2.- Monthly census of the cattle egret in roosts located in Cantabria during 2014. 
el solapamiento de las áreas de campeo de los diferentes individuos de ambas dan como resultado la presencia de garcillas bueyeras en la práctica totalidad de la franja costera de Cantabria.

En cuanto a los dormideros se refiere, todos se ubicaron en humedales a excepción del situado en el Zoológico de Santillana del Mar, no ligado a ninguna masa de agua. El tipo de humedal utilizado más frecuentemente fue la laguna de agua dulce, donde se localizaron cinco de los 18 dormideros prospectados. Tanto en lagunas salobres como en rías encontramos tres dormideros respectivamente. En marismas y cauces fluviales, se encontraron dos. Por último uno de los dormideros se ubicó en una bahía y otro en un islote costero. Con respecto al tipo de sustrato, el más comúnmente utilizado fue el arbóreo (11 dormideros). El resto se ubicaron en vegetación arbustiva (tres dormideros), carrizal (tres dormideros), estructuras artificiales de origen antrópico no destinadas a las aves (e.g. gasoducto en la bahía de Santander; tres dormideros) o bien en rocas o pequeñas isletas naturales sin vegetación dentro de los propios humedales (tres dormideros). En los dormideros de Marismas Blancas en Astillero, Marismas de Alday en Camargo, Finca de La Remonta en Santander, ría de San Vicente de la Barquera y Marismas de Joyel en Arnuero, las aves utilizaron diferentes tipos de sustratos para pernoctar a lo largo del estudio, de ahí que sumando todos los posaderos indicados, el resultado sea mayor de dieciocho, que es el total de dormideros estudiados.

Se apreciaron diferencias de comportamiento entre los dormideros definidos como principales y aquellos clasificados como secundarios. En el primer caso, los primeros bandos se instalaron previamente en la periferia del dormidero, en lugar de entrar directamente a éste. Una vez congregado un grupo numeroso (entre 200 y 1250 ejemplares, dependiendo del lugar) las aves esperaban hasta que uno de los nuevos grupos que acudían, se posaba en la zona concreta donde iban a pasar la noche. Después, todos los individuos ubicados en la zona de posadero provisional entraban simultáneamente al dormidero. Este comportamiento no se observó en los dormideros secundarios. Otra diferencia la encontramos en la forma de llegada, ya que en los dormideros principales la entrada de bandos normalmente tenía una duración de entre 30 y 45 minutos, pudiendo prolongarse de manera excepcional hasta la hora y media. En los dormideros secundarios la entrada de aves se produjo en forma de uno o dos bandos que solían llegar con una diferencia de no más de 10 minutos entre sí.

\section{Discusión}

La población de garcillas bueyeras viene experimentando un aumento continuado desde que las primeras aves de esta especie se moviesen libremente por Cantabria a mediados de la década de los 90. El foco de dispersión fue el zoológico de Santillana del Mar (GarcíaFernández, 2000) y desde allí comenzaron a campear hacia el este de la provincia, observándose a día de hoy en toda su franja costera. 


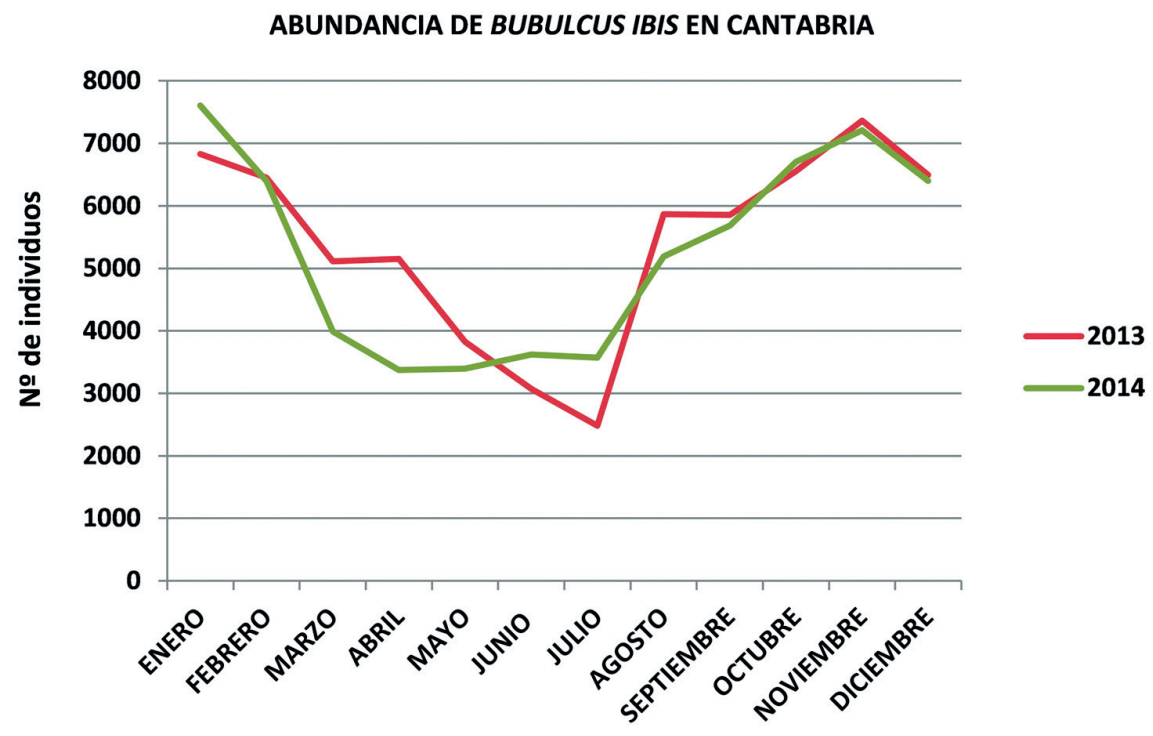

Fig.2.- Evolución mensual del número de garcillas bueyeras censadas en los dormideros de Cantabria en 2013 y 2014.

Fig. 2.- Monthly evolution of the number of cattle egrets counted in the roosts in Cantabria in 2013 and 2014.

De acuerdo con los patrones de variación temporal en la abundancia obtenidos en nuestro estudio, al menos una fracción de la población reproductora sería sedentaria, lo que se sustentaría en la presencia en el mes de noviembre de un ave nacida en Noja, y en el hecho de que la abundancia a partir de agosto aumente mes a mes hasta noviembre sin experimentar descensos, lo que hace pensar que al menos una fracción de esta población reproductora permanece durante todo el año.

Por otro lado, existiría otro contingente invernante proveniente de otras regiones, que alcanza en el mes de noviembre la máxima abundancia, para descender en el mes de diciembre y de nuevo aumentar en enero. Este hecho contrasta con otros estudios realizados en poblaciones andaluzas donde, tras alcanzar los máximos en diciembre, el descenso poblacional tiene lugar en enero (Rodríguez y Tarragona, 1995; González Cachinero, 2006). Durante la época invernal su distribución se amplía a algunas localidades de la costa del País Vasco y de Asturias, donde ha habido recuperaciones de anillas de ejemplares nacidos en la colonia de Noja (SEO/BirdLife, delegación territorial) y nos da una idea de su expansión hacia el oeste a lo largo de la franja costera cantábrica.

La población invernante para Cantabria, estimada en una media de 4.229 aves en el periodo 2007-2010 (Garrido et al., 2012) ha aumentado en los últimos años con una media de $6.856,5$ individuos en el periodo de estudio, si bien parece observarse un estancamiento 
de su crecimiento. La variabilidad en el número de ejemplares detectada en éste periodo (Error Estándar de la Media), ha resultado ser menor que el obtenido en el periodo reproductor, pese a que la abundancia invernal es mucho mayor. Durante los meses de marzo y abril, al inicio de la época de cría, se han registrado abundancias de más de 5.000 individuos, posiblemente por la presencia de las últimas aves invernantes y ejemplares migrantes que sedimentan en la zona en su viaje migratorio. Posteriormente, en el mes de julio, y quizás influenciado por el mayor éxito/fracaso en la reproducción, el número de aves puede caer a tan solo 2.500 individuos. Estos fuertes cambios en la abundancia no se dan durante el invierno, cuando el número de garcillas ha permanecido entre los 6.400 y los 7.600 ejemplares en el periodo octubre-febrero a lo largo del estudio.

Coincidiendo con el trabajo de Garrido et al. (2012), el sustrato en el que se ubicaron la mayor parte de los dormideros a nivel nacional fue la vegetación arbórea. Por el contrario, ninguno se ubicó en pastizales o herbazales, a pesar de que éste es el sustrato sobre el que se ubica el 29\% de los dormideros invernales a nivel nacional, según el mismo trabajo.

El hecho de que tan solo el dormidero ubicado en el islote de San Pedruco, en Noja, haya sido ocupado durante los doce meses del año, nos da una idea de la alta movilidad de la especie a nivel local. Se trata de un islote rocoso que alberga una de las dos únicas colonias de cría existentes en el cantábrico. En los años 2012 y 2013 fue abandonado durante el período invernal, pero en 2014 fue utilizado durante todo el año. No se han observado perturbaciones de ningún tipo ni en éste, ni en ninguno de los demás dormideros, por lo que descartamos las molestias como motivo de cambio de una ubicación.

Pese a ser escasos los estudios sobre la invernada de esta especie en el Paleártico, sabemos que una buena parte del contingente reproductor de la Camarga, en la costa mediterránea francesa, pasa el invierno en las costas mediterráneas españolas (Pineau et al., 1992) y en general, aproximadamente un tercio de la población reproductora de todo Francia pasa el invierno en España (Dubois et al, 2008). Igualmente, en Reino Unido existe una población invernante y otra reproductora (RSPB, BTO, 2008). Las bajas temperaturas invernales son un factor de mortandad para estas y otras ardeidas (North, 1979; Pineau, 1992; Fleury com. pers., 2014) por lo que parece lógico pensar que lleguen a las costas del cantábrico aves que se han reproducido en latitudes más norteñas dentro de la vertiente atlántica.

Por otro lado, en los años posteriores al escape del núcleo zoológico, teniendo en cuenta la expansión a nivel peninsular, también habrían llegado ejemplares a través del valle del Ebro, ya que los sistemas montañosos existentes en la frontera con Castilla y León actúan de barrera natural para la especie (Sarasa et al., 1997). Con todo ello, a día de hoy pueden observarse garcillas bueyeras en gran parte del territorio cántabro, ya que son capaces de trasladarse más de 60 km desde la colonia y/o dormidero en busca de alimento (Cramp y Simmons, 1977) si bien, es en la franja costera donde se han establecido, posiblemente por el clima más benigno allí presente en comparación con la mitad sur de la provincia.

Con todo ello, no cabe duda de que las garcillas bueyeras han pasado a formar parte de la avifauna cantábrica en apenas 20 años donde, con carácter invernante y residente, se han asentado con facilidad en la mitad norte de la provincia. 


\section{Agradecimientos}

En primer lugar, nuestros más sinceros agradecimientos a Aitor Rincón por su esencial colaboración en los censos mensuales. A Felipe González y Pablo Díaz-Vega por la información aportada sobre algunos dormideros. Aitor Galarza y dos revisores anónimos aportaron comentarios sobre versiones iniciales del manuscrito.

\section{Bibliografía}

- Tahillo, M., Alonso, I. 1998. Fauna de Cantabria. Aves Acuáticas y Marinas. Ed. Cantabria Tradicional.

- Barrett, A. 15 January 2008. Flying in ... to make new friends down on the farm. Irish Independent 15 Jaunary 2008. Available from: http://www.independent.ie/national-news/flying-into-make-new-friends-down-on-the-farm-1266360.html.

- Cramp, S. y Simmons, K.E.L. (Eds.) 1997. The Birds of the Western Paleartic, Vol.1. Oxford University Press. Oxford.

- De Juana, E., García, E. 2015. The Birds of the Iberian Península. Bloomsbury Eds.

- Dubois, P.J., Le Maréchal, P.L., Olioso, G., Yésou, P. 2008. Nouvel inventaire des oiseaux de France. Delachaux et Niestlé, Paris.

- Fleury, A. 2014. (Com.pers.) Parc Ornithologique du Teich. France.

- García-Fernández, J. 2000. La expansión de la Garcilla Bueyera (Bubulcus ibis) en el noreste de la Península Ibérica. Rev. Ecología 14: 269-273

- Garrido, J.R., Molina, B., Del Moral, J.C. (Eds.) 2012. Las garzas en España, población reproductora e invernante en 2010-2011 y método de censo. SEO/BirdLife. Madrid.

- Guardiola Gómez, A. 1999. Plan de manejo de la garcilla bueyera (Bubulcus ibis) en la Región de Murcia. En: Planes de Gestión de Ardeideas y Anátidas Amenazadas de la Rregión de Murcia. M.A. Sánchez Sánchez, E. Díez de Revenga Martínez (Eds.). AMBIENTAL, S.L. para la Dirección General del Medio Natural, Consejería de Medio Ambiente, Agricultura y Agua.

- González Cachinero, J.M. 2006. Winter census of cattle egret (Bubulcus ibis Linnaeus, 1758) in the province of Granada (SE Spain) winter of 2005-2006. Acta Granatense 4/5:61-65.

- Krebs, E.A., Riven-Ramsey, D., Hunte, W. 1994. The Colonization of Barbados by Cattle Egrets (Bubulcus ibis) 1956-1990. Colonial Waterbirds (Waterbird Society) 17 (1): 86-90.

- Maddock, M. 1990. Cattle Egrets: South to Tasmania and New Zealand for the winter. Notornis 37 (1): 1-23.

- Martí, R., Del Moral, J.C. 2003 (Eds.). Atlas de las Aves reproductoras en España. Dirección General de Conservación de la Naturaleza-SEO/BirdLife. Madrid.

- Martínez-Vilalta, A., Motis, A. 1992. Family Ardeidae (Herons). En: Handbook of the Birds of the World. Volume 1: Ostrich to Ducks. J. del Hoyo, A. Elliot, A., J. Sargatal, J.: 401-402. Lynx Edicions. 
- Nightingale, B., Dempsey, E. 2008. Recent reports. British Birds 101(2): 108.

- North, P.M. 1979. Relating Grey Heron survival rates to winter weather conditions. Bird Study $26: 23-28$.

- Pineau, O., Hafner, H., Wallace, J.P. 1992. The effects of winter climate on the size of the cattle egret (Bubulcus ibis I.) population in the camargue. Rev. Ecol. Terre Vie 47.

- Rodríguez, E. y Tarragona, F. 1995. La Garcilla Bueyera en el delta del Guadalfeo. El Colorín 1: 30-35.

- RSPB, BTO, 2008. Cattle egret in The United Kingdom.

- Sarasa, C.G., Garrido, J.R., Bartolomé, J., Igual, J.M., Fernández-Cruz, M. 1997. Movimientos poblacionales y tasa de mortalidad de la Garcilla Bueyera (Bubulcus ibis, L. 1758) en la Península Ibérica. En: Actas de las XII Jornadas Ornitológicas Españolas. J. Manrique, A. Sánchez, F. Suárez y M. Yanes (Coords.): 239-244. Instituto de Estudios Almerienses. Diputación de Almería. Almería.

- Silva, M.P., Coria, N.E., Favero, M., Casaux, R.J. 1995. New Records of Cattle Egret Bubulcus ibis, Blacknecked Swan Cygnus melancoryhyphus and White-rumped Sandpiper Calidris fuscicollis from the South Shetland Islands, Antarctica. Marine Ornithology 23: 65-66.

\section{है}

- Fecha de recepción/Date of reception: 10-10-2016

- Fecha de aceptación/Date of acceptance: 06-03-2017 